\title{
Charge density and electric charge in quantum electrodynamics
}

\author{
G. Morchio, \\ Dip. di Fisica, Universita' di Pisa and INFN, Pisa, Italy \\ F. Strocchi, \\ Scuola Normale Superiore and INFN, Pisa, Italy
}

\begin{abstract}
The convergence of integrals over charge densities is discussed in relation with the problem of electric charge and (non local) charged states in Quantum Electrodynamics (QED). Delicate, but physically relevant, mathematical points like the domain dependence of local charges as quadratic forms and the time smearing needed for strong convergence of integrals of charge densities are analyzed. The results are applied to QED and the choice of time smearing is shown to be crucial for the removal of the vacuum polarization effects responsible for the time dependence of the charge (Swieca phenomenon). The possibility of constructing physical charged states in the Feynman-GuptaBleuler gauge as limits of local state vectors is discussed, compatibly with the vanishing of the Gauss charge on local states. A modification by a gauge term of the Dirac exponential factor which yields the physical Coulomb fields from the Feynman-Gupta-Bleuler fields is shown to remove the infrared divergence of scalar products of local and physical charged states, allowing for a construction of physical charged fields with well defined correlation functions with local fields.
\end{abstract}




\section{Introduction}

The simple relation between charge density and electric charge in classical electrodynamics does not extend trivially to the quantum case, because of problems due to vacuum polarization and infinite volume integration.

Quite generally, the relation between local charges and global conserved charges has been extensively discussed in the seventies, in relation with the proof of the Goldstone theorem [1, 2, 3, 4] and it has become standard wisdom in the quantum field theory (QFT) framework in which all the relevant information are carried by the local states.

The problem changes substantially if the relevant charged states are non local, as it is the case of Quantum Electrodynamics(QED) [5]. As a consequence, one cannot rely on the standard strategy of controlling the convergence of local charges on the domain of local states, and in fact the limit of local charges, as quadratic forms, crucially depends on the domain which is considered. Moreover, as discussed by Swieca [7, on the charged states obtained by applying Coulomb fields to the vacuum, the local charge given by the integral of the density with the standard smearing in space and time does not converge to the electric charge and its limit is even time dependent.

This difficulty requires an analysis of the convergence of suitably timesmeared integrals of the charge density; as we shall see, not only the standard time smearing does not work, but also Requardt's space-time smearing prescription 4] requires a modification in order to obtain the correct result for the renormalized charge. Actually, the basic point is the control of the construction of charged states, which is related to the infrared problem and is a deep non perturbative problem, both in the general algebraic approach and in the approach which uses fields operators [6].

Even in perturbation theory a rigorous control on the construction of charged states is far from trivial. In the (positive) Coulomb gauge the (non local) charged fields are difficult to handle [8] and the standard strategy is to use a local formulation at the expense of positivity, as in the FeynmanGupta-Bleuler gauge. In this case, the charged states should be the obtained by an appropriate construction in terms of local (unphysical) states. Such a possibility has been advocated by Dirac and Symanzik [9, 8, who proposed explicit formulas for non local charged (Coulomb) fields in terms of the local Feynman-Gupta-Bleuler fields. Such a construction, which involves non trivial ultraviolet and infrared problems has recently been refined by Steinmann [10, 13] on the basis of a perturbative expansion. 
An important issue is whether the above states can be constructed only in terms of expectations of the observables or they exist as vectors in a space in which local states are dense. In the latter case, the control of limits of local states requires a topology and the topology defined by the Wightman functions of the local fields is too weak to give a unique space; thus, the possibility of reaching the physical charged states, characterized by a Coulomb delocalization, depends on the choice of a topology. For example, the implicit use of the standard (Krein) metric on the asymptotic fields $A_{\mu}^{a s}$ excludes the presence of charged states in the corresponding physical space, as pointed out by Zwanziger in his investigations on the infrared problem in QED [11. A possible non perturbative construction of physical charged states as limits of local states was discussed in Ref. [12, with the use of a Hilbert-Krein topology which takes into account the effects of the infrared problem. In our opinion, the non uniqueness of such Hilbert-Krein majorant topologies, which are associated to the Wightman functions of the local fields in order to obtain weakly complete inner product spaces of states, should not be regarded as a mathematical oddness, being related to the allowed large distance behaviour or "boundary conditions" at infinity.

The possibility of constructing physical charged states as limits of local state vectors in a weak topology has been recently denied [13] on the basis of an argument by which the local Gauss charge, corresponding to the integral of div $E$, vanishes on the local states and therefore on any weak closure of them; thus no weak closure of the local states could contain physical charged states. A main conclusion of our analysis is that the assumptions involved in the argument underestimate the delicate rôle of such topologies for the convergence of local charges in QED.

In view of the problems which arise in QED, in Section 2 we discuss in general charges defined as limits of quadratic forms, their crucial dependence on the domain and their relation to global charge operators; in particular, attention is paid to the case in which the relevant domains arise by applying non local field operators to the vacuum.

In Section 3 we consider the problem of weak convergence of local charges, which is shown to be very relevant for the Steinmann argument. Strong convergence on the vacuum is shown to be a general consequence of a stronger version of Requardt's theorem, which also allows for an improved time smearing procedure, necessary for obtaining the correct value of the charge on Coulomb charged states. Such a time smearing procedure avoids the time dependence effects due to vacuum polarization while preserving the correct 
value of the charge.

In Section 4, we discuss convergence of Gauss local charges on physical charged states, on the basis of the standard local formulations of QED, the Feynman-Gupta-Bleuler gauge. Quite generally, independently of the use of a Hilbert-Krein topology, it is shown that the construction of physical charged state vectors as limits of local states in a weak topology is incompatible with convergence, in the same weak topology, of the Gauss local charges, even with a time smearing a la Requardt, on local states. A simple model is discussed which mimics the relation between charge density and charge in QED and displays the compatibility between the vanishing of the Gauss charge on a dense domain of local states and its strong convergence to a non zero electric charge on the physical space. In Section 5 we compare the construction of physical charged states of Ref.[12] with the DSS construction analyzed by Steinmann [10, 13]. We show that the infrared divergence in the matrix elements of physical charged states with local states is avoided by the modified DSS exponential used in Ref. [12], which only differs from the standard factor by a gauge term. In this way one removes the obstruction pointed out by Steinmann [13] as an argument for the impossibility of constructing physical charged fields with well defined correlation functions with local fields.

\section{Charges as limits of quadratic forms}

The analysis of the charge operator in QED presents subtle features arising from the Coulomb delocalization of charged states [5, 12. It is therefore convenient to start by an analysis of charges as integrals over a local density on general (not necessarily local) domains.

In this section we shall show that i) charges defined as limits of quadratic forms $Q_{R}$ on dense domains $\mathcal{D} \times \mathcal{D}$ in general (including the quantum field theory case) crucially depend on the domain; e.g. $Q_{R}$ may converge to zero on $\mathcal{D} \times \mathcal{D}$ and have a non zero limit on $\mathcal{D}_{1} \times \mathcal{D}_{1}$, if $\mathcal{D} \cap \mathcal{D}_{1}$ is not dense, ii) such a phenomenon cannot occur if $Q_{R}$ converges weakly on $\mathcal{D}$ and on $\mathcal{D}_{1}$.

Quite generally, in quantum field theory the problem of associating an (unbroken) charge $Q$ to the integral over a local density

$$
Q_{R}=\int_{|x| \leq R} d x j_{0}(x, 0), \quad \partial_{\mu} j^{\mu}=0,
$$


is delicate and deserves special attention. Intuitively, one thinks of defining a state of charge $q$, as satisfying

$$
Q \Psi=\lim _{R \rightarrow \infty} Q_{R} \Psi=q \Psi
$$

but as emphasized by Schoer and Stichel [14, the limit does not exist as a weak limit, even if some smearing in time is made with $\alpha\left(x_{0}\right), \alpha \in \mathcal{D}(\mathbf{R})$ and even if $\Psi$ is a local state, briefly $\Psi \in \mathcal{D}_{0}$. In the latter case, the limit exists [3] as a sesquilinear form on $\mathcal{D}_{0} \times \mathcal{D}_{0}$

$$
\lim _{R \rightarrow \infty}\left(\Phi, Q_{R} \Psi\right)=Q(\Phi, \Psi), \Phi, \Psi \in \mathcal{D}_{0} .
$$

Furthermore, if $Q_{R}$ defines an unbroken symmetry on the local fields the limit sesquilinear form defines an (hermitean) operator $Q$ on $\mathcal{D}_{0}$.

\section{i) Domains and limits of quadratic forms}

In general, the limit of hermitean operators $Q_{R}$ as forms on domains $\mathcal{D} \times \mathcal{D}$, crucially depends on the domain $\mathcal{D}$, in particular, the limit on $\mathcal{D} \times \mathcal{D}$ does not constrain the limit on $\mathcal{D}_{1} \times \mathcal{D}_{1}, \mathcal{D}_{1} \neq \mathcal{D}$.

Such a domain dependence in general persists, as shown by the example below, even if $Q_{R}$ converges to an hermitean sesquilinear form $Q$ on $\mathcal{D} \times \mathcal{D}$ satisfying the boundedness condition

$$
|Q(\Phi, \Psi)| \leq C_{\Psi}\|\Phi\|, \forall \Phi, \Psi \in \mathcal{D}
$$

and therefore identifies an (hermitean) operator $Q$ with domain $\mathcal{D}$. Furthermore, even if eq. $(2,1)$ holds, it is not at all guaranteed that, $\forall \chi$,

$$
(\chi, Q \Psi)=\lim _{R \rightarrow \infty}\left(\chi, Q_{R} \Psi\right), \quad \forall \Psi \in \mathcal{D} .
$$

In fact, such an equation means that $Q_{R} \mathcal{D}$ converge weakly. By the convergence of $Q_{R}$ on $\mathcal{D} \times \mathcal{D}$, weak convergence of $Q_{R} \mathcal{D}$ is equivalent to the boundedness of the norms $\left\|Q_{R} \Psi\right\|$, for each fixed $\Psi \in \mathcal{D}$.

In particular, as shown by the example below, even if $\left(\Phi, Q_{R} \Psi\right)$ converges to zero $\forall \Phi, \Psi \in \mathcal{D}$, one cannot conclude that $\forall \chi, \lim _{R \rightarrow \infty}\left(\chi, Q_{R} \Psi\right)=0$. (This also shows that the failure of eq.(2.2) does not depend on $Q_{R}$ converging to an unbounded or a bounded operator.)

The general phenomenon is that, if $Q_{R}$ converge to an operator $Q_{0}$ on $\mathcal{D}_{0} \times \mathcal{D}_{0}$ and to an operator $Q_{1}$ on $\mathcal{D}_{1} \times \mathcal{D}_{1}$, the two operators $Q_{0}$ and $Q_{1}$ are in general not related, in the sense of the following 
Definition 2.1 Two densely defined hermitean operators $Q_{0}, Q_{1}$ are said to be related if there is an hermitean operator $Q$ of which $Q_{0}$ and $Q_{1}$ are restrictions. They will be said to be weakly related if there is a densely defined hermitean operator $Q_{2}$ to which both $Q_{0}$ and $Q_{1}$ are related.

The above relations are symmetric and the second notion is strictly weaker, since e.g. different self adjoint extensions of an hermitean operator are not restrictions of the same hermitean operator. An example of limits of quadratic forms which define not weakly related operators is given below.

Example. Let us consider $L^{2}([0, \pi], d x), \mathcal{D}_{0} \equiv$ the space of $C^{\infty}$ functions vanishing at the origin, $\mathcal{D}_{1} \equiv$ the linear span of $f_{1}(x) \equiv 1$ and $f_{n}(x) \equiv$ $\sin n x-\alpha_{n} \sin x, n \geq 2, \alpha_{n} \equiv 1 / 2 \int_{0}^{\pi} \sin n x$, so that $\left(f_{1}, f_{n}\right)=0$.

Clearly, both $\mathcal{D}_{0}$ and $\mathcal{D}_{1}$ are dense domains; in fact, if $f$ is orthogonal to $\mathcal{D}_{1}$ one has

$$
c_{n} \equiv(f, \sin n x)=\alpha_{n}(f, \sin x)=\alpha_{n} c_{1}, n \geq 2 .
$$

Furthermore

$$
0=(\pi / 2) \int_{0}^{\pi} d x f(x)=\sum_{n \geq 1} c_{n} \int_{0}^{\pi} d x \sin n x=2\left(\sum_{n \geq 2} \alpha_{n}^{2} c_{1}+c_{1}\right)
$$

implies $c_{1}=0$, i.e. $f=0$. Now, let $Q_{R}$ be the multiplication operator by a regular function $q_{R}(x)$ converging to $\delta(x)$ as a distribution; then

$$
\left(\mathcal{D}_{0}, Q_{R} \mathcal{D}_{0}\right) \rightarrow 0, \quad\left(\mathcal{D}_{1}, Q_{R} \mathcal{D}_{1}\right) \rightarrow\left(\mathcal{D}_{1}, P_{1} \mathcal{D}_{1}\right) \neq 0
$$

with $P_{1}$ the projection on $f_{1}$. Thus, the limits of the hermitean operators $Q_{R}$ define two bounded operators which are not even weakly related.

Convergence of $Q_{R}$ on $\mathcal{D}_{0} \times \mathcal{D}_{0}$ to an operator $Q_{0}$ constrains convergence to an operator on any domain $\mathcal{D} \times \mathcal{D}$, such that $\mathcal{D} \cap \mathcal{D}_{0}$ is dense.

Proposition 2.1 Let the hermitean operators $Q_{R}$ converge to an operator $Q_{0}$ on $\mathcal{D}_{0} \times \mathcal{D}_{0}$ and to an operator $Q_{1}$ on $\mathcal{D}_{1} \times \mathcal{D}_{1}$;

i) if $\mathcal{D}_{1} \cap \mathcal{D}_{0}$ is dense, then $Q_{0}$ and $Q_{1}$ are weakly related

ii) if $\mathcal{D}_{1} \supset \mathcal{D}_{0}$, then $Q_{0}$ and $Q_{1}$ are related

iii) in both cases, if $Q_{0}$ is essentially selfadjoint on $\mathcal{D}_{1} \cap \mathcal{D}_{0}$, then $Q_{1}$ is contained in the closure of $Q_{0}$

iv) if $Q_{0}$ and $Q_{1}$ are not related, then $Q_{R}$ does not converge to an operator $Q$ on $\mathcal{D} \times \mathcal{D}, \mathcal{D}=\mathcal{D}_{0}+\mathcal{D}_{1}$. 
Proof The hermiticity of $Q_{R}$ implies that both $Q_{i}, i=0,1$ are densely defined hermitean operators and so is their restriction $Q$ to $\mathcal{D}_{1} \cap \mathcal{D}_{0}$. In case ii) $Q_{1}$ extends $Q_{0}$, in case i) $Q_{1}$ and $Q_{0}$ extend $Q$. If $Q_{R}$ converge to $Q$ on $\mathcal{D} \times \mathcal{D}$, both $Q_{0}$ and $Q_{1}$ are restrictions of $Q$, so that $Q_{0}$ and $Q_{1}$ are related.

Proposition 2.2 If both $Q_{R} \mathcal{D}_{0}$ and $Q_{R} \mathcal{D}_{1}$ converge weakly, then the two limits define hermitean operators $Q_{0}$ and $Q_{1}$ which are related

Proof Hermiticity of the limit forms follows from that of $Q_{R}$ and the existence of weak limits implies that the limit forms define operators $Q_{0}$ on $\mathcal{D}_{0}$ and $Q_{1}$ on $\mathcal{D}_{1}$. The weak limit of $Q_{R}$ exists also on $\mathcal{D}_{0}+\mathcal{D}_{1}$ and by the same argument defines an hermitean operator $Q$ which extends $Q_{0}$ and $Q_{1}$.

As a result, if $Q_{0}$ is essentially selfadjoint, $Q_{1}$ is contained in its closure and in particular if $Q_{0}=0$, also $Q_{1}=0$, in other terms if $Q_{R} \mathcal{D}_{0}$ converges to zero weakly and $Q_{R} \mathcal{D}_{1}$ converges weakly, then $Q_{1}=0$.

\section{ii) Convergence of local charges in quantum field theory}

A general situation which occurs in quantum field theory is described in terms of translational invariant (field) algebras $\mathcal{A}_{0}, \mathcal{A}_{1}$, a (unique translationally invariant) cyclic vector $\Psi_{0}$, domains

$$
\mathcal{D}_{0}=\mathcal{A}_{0} \Psi_{0}, \quad \mathcal{D}_{1}=\mathcal{A}_{1} \Psi_{0},
$$

and local hermitean charges $Q_{R}$, with domains containing $\mathcal{D}_{0}$ and $\mathcal{D}_{1}$ and with $\left(\Psi_{0}, Q_{R} \Psi_{0}\right)=0$. In general, $Q_{R}$ is the integral of the zero component of a local conserved (operator valued tempered distribution) current $j_{\mu}$ with suitable smearing:

$$
\begin{gathered}
Q_{R}=\int d^{4} x j_{0}\left(\mathbf{x}, x_{0}\right) f_{R}(\mathbf{x}) \alpha\left(x_{0}\right)=j_{0}\left(f_{R} \alpha\right), \\
f_{R}(x)=f(|x| / R) \in \mathcal{D}\left(\mathbf{R}^{3}\right), f(x)=1, \text { if }|x| \leq 1, f(x)=0, \text { if }|x| \geq 2, \\
\alpha \in \mathcal{D}(\mathbf{R}), \operatorname{supp} \alpha \subset[-a, a], a<1, \int d t \alpha(t)=1 .
\end{gathered}
$$

If $\mathcal{A}_{0}$ is a local (field) algebra and $\left(\mathcal{D}_{0}, Q_{R} \mathcal{D}_{0}\right)$ converges as $R \rightarrow \infty$, the limit defines an operator $Q_{0}$ iff

$$
\lim _{R \rightarrow \infty}\left(\Psi_{0},\left[Q_{R}, \mathcal{A}_{0}\right] \Psi_{0}\right)=0,
$$

equivalently [3] iff

$$
\lim _{R \rightarrow \infty}\left(\mathcal{D}_{0}, Q_{R} \Psi_{0}\right)=0 .
$$


Non local algebras may be relevant in the discussion of non local states, e.g. asymptotic states, or charged states in the Coulomb gauge; a local and a non local field algebra, $\mathcal{A}_{0}$ and $\mathcal{A}_{1}$, occur in the construction of charged states in QED.

Proposition 2.3 Let $\mathcal{D}=\mathcal{A} \Psi_{0}, \mathcal{A}$ an algebra invariant under translations; if on $\mathcal{D} \times \mathcal{D}, Q_{R}$ converge to an operator $Q$, then

$$
\lim _{R \rightarrow \infty}\left(\mathcal{D}, Q_{R} \Psi_{0}\right)=0
$$

Proof The spectral representations of the space translations gives

$$
\left((U(\mathbf{a})-1)^{4} A \Psi_{0}, Q_{R} \Psi_{0}\right)=\int d J_{A}(\mathbf{k})\left(e^{i \mathbf{k} \cdot \mathbf{a}}-1\right)^{4} R^{3} \tilde{f}(R \mathbf{k}), \forall A \in \mathcal{A}
$$

where $d J_{A}(\mathbf{k})=\int d J_{A}\left(\mathbf{k}, k_{0}\right) \tilde{\alpha}\left(k_{0}\right)$ is a complex measure of polynomial growth. Now, since for any polynomial $P(\mathbf{k})$

$$
\left|\left(e^{i \mathbf{k} \cdot \mathbf{a}}-1\right)^{4} R^{3} \tilde{f}(R \mathbf{k}) P(\mathbf{k})\right| \leq \frac{|R \mathbf{k} \cdot \mathbf{a}|^{4}}{R}|\tilde{f}(R \mathbf{k}) P(R \mathbf{k})|\left|\frac{P(\mathbf{k})}{P(R \mathbf{k})}\right| \leq \frac{C}{R} \rightarrow 0,
$$

in the limit $R \rightarrow \infty$, the r.h.s. of eq.(2.6) converges to zero and therefore, by the density of $\mathcal{D}$, one has

$$
(U(\mathbf{a})-1)^{4} Q \Psi_{0}=0, \quad \forall \mathbf{a} .
$$

Then, since $U(\mathbf{a})-1$ is a normal operator, it follows that $(U(\mathbf{a})-1) Q \Psi_{0}=0$, and by the uniqueness of the translationally invariant state $Q \Psi_{0}=\lambda \Psi_{0}$; actually $Q \Psi_{0}=0$, because $\left(\Psi_{0}, j_{0} \Psi_{0}\right)=0$.

Thus, under the same assumptions, one has that the charge $Q^{\prime}$ defined in terms of the limit of the commutator [15], coincides with $Q$, i.e.

$$
\left(\mathcal{D}, Q^{\prime} A \Psi_{0}\right) \equiv \lim _{R \rightarrow \infty}\left(\mathcal{D},\left[Q_{R}, A\right] \Psi_{0}\right)=\lim _{R \rightarrow \infty}\left(\mathcal{D}, Q_{R} A \Psi_{0}\right)
$$

The domain dependence of charge operators obtained as limits of quadratic forms appears also in the above quantum field theory framework. In particular, as a result of Proposition 2.1, if $Q_{R}$ converges to zero on $\mathcal{D}_{0} \times \mathcal{D}_{0}$, the convergence to a non zero operator on $\mathcal{D}_{1} \times \mathcal{D}_{1}$ is excluded if $\mathcal{D}_{1} \cap \mathcal{D}_{0}$ is dense, but may be allowed if $\mathcal{D}_{1} \cap \mathcal{D}_{0}$ is not dense, even if $\Psi_{0} \in \mathcal{D}_{1} \cap \mathcal{D}_{0}$.

Such features are illustrated and displayed by the following Example. 
Example. Let $\phi$ be a massless scalar field, $\psi$ a free Dirac field, $\mathcal{A}_{0}$ the algebra generated by $\partial_{i} \phi, i=1,2,3$ and by $\psi$ and $\mathcal{A}$ the algebra generated by $\partial_{i} \phi$ and by

$$
\begin{gathered}
\psi_{d}(x)=\psi(x) U(x), \quad U(x)=e^{i \phi\left(f_{x}\right)} \\
\phi\left(f_{x}\right)=\int d y \phi(y) f(y-x), \quad f \in \mathcal{D}\left(\mathbf{R}^{4}\right), \quad \int d x f(x)=1 .
\end{gathered}
$$

Then we consider the local charges

$$
\begin{gathered}
Q_{R}{ }^{\phi} \equiv \partial_{0} \phi\left(f_{R} \alpha,\right), \quad Q_{R}{ }^{\psi} \equiv j_{0}\left(f_{R} \alpha\right), \quad j_{\mu}(x)=: \bar{\psi} \gamma_{\mu} \psi:, \\
Q_{R}=Q_{R}{ }^{\psi}+Q_{R}{ }^{\phi}
\end{gathered}
$$

and the Fock representation of $\psi, \phi$, with Fock vacuum $\Psi_{0}$. Since by locality

$$
\lim _{R \rightarrow \infty}\left[Q_{R}^{\phi}, \mathcal{A}_{0}\right]=0, \lim _{R \rightarrow \infty}\left(\mathcal{D}_{0}, Q_{R} \Psi_{0}\right)=0,
$$

we have

$$
\lim _{R \rightarrow \infty}\left(\mathcal{D}_{0}, Q_{R} \mathcal{D}_{0}\right)=\left(\mathcal{D}_{0}, Q^{\psi} \mathcal{D}_{0}\right),
$$

where $Q^{\psi}$ is the unbroken fermionic charge. On the other hand, since $\lim _{R}\left[Q_{R}, \psi_{d}(g)\right]=0$ we have

$$
\lim _{R \rightarrow \infty}\left(\mathcal{D}, Q_{R} \mathcal{D}\right)=0 .
$$

In conclusion $Q_{R}$ converge to the unbroken fermionic charge on $\mathcal{D}_{0} \times \mathcal{D}_{0}$ and to the zero charge on $\mathcal{D} \times \mathcal{D}$.

It is worthwhile to note that the limit of the operators $Q_{R}$ does not define an operator on $\mathcal{D}_{\text {ext }} \times \mathcal{D}_{\text {ext }}$, where $\mathcal{D}_{\text {ext }}=\mathcal{D}_{0}+\mathcal{D}$, (since the corresponding bilinear form is discontinuous on the left). Moreover, one has a symmetry breaking condition on the algebra $\mathcal{A}_{\text {ext }}$ generated by $\mathcal{A}_{0}$ and $\mathcal{A}_{1}$ : $\left[Q_{R}, \mathcal{A}_{e x t}\right] \Psi_{0}$ converges weakly (actually strongly) and

$$
\lim _{R \rightarrow \infty}\left(\Psi_{0},\left[Q_{R}, \psi^{\dagger} \psi_{d}\right] \Psi_{0}\right) \neq 0 .
$$

This fact is actually a consequence of $Q_{0}$ and $Q_{1}$ being not related. In general if $Q_{R}$ converges on $\mathcal{D}_{i} \times \mathcal{D}_{i}, i=0,1$ to operators $Q_{i}$ which are not related, then, for the algebra $\mathcal{A}$ generated by $\mathcal{A}_{0}$ and $\mathcal{A}_{1}$, one cannot have both weak convergence of $\left[Q_{R}, \mathcal{A}\right] \Psi_{0}$ and

$$
\lim _{R \rightarrow \infty}\left(\Psi_{0},\left[Q_{R}, \mathcal{A}\right] \Psi_{0}\right)=0 .
$$


In fact, by eq.(2.6),

$$
\left(\mathcal{D}_{i}, Q_{i} A \Psi_{0}\right) \equiv \lim _{R \rightarrow \infty}\left(\mathcal{D}_{i},\left[Q_{R}, A\right] \Psi_{0}\right), \quad \forall A \in \mathcal{A}_{i} .
$$

Now, if eq.(2.9) holds, by a standard argument [15] one gets an hermitean operator $Q$ on $\mathcal{D} \equiv \mathcal{A} \Psi_{0}$, which extends $Q_{0}$ and $Q_{1}$, in contrast with their being not related.

\section{Convergence of time smeared integral of charge density. The vacuum sector of QED}

In this section we discuss weak and strong convergence of local charges, in particular in the vacuum sector of QED.

As found by Requardt [2], the weak limit of $Q_{R}$ on local states can be obtained under general conditions by a suitable time smearing of the charge density, namely by considering, with $f_{R}, \alpha$ as in eq.(2.3),

$$
Q_{R} \equiv j_{0}\left(f_{R} \alpha_{R}\right), \quad \alpha_{R}\left(x_{0}\right) \equiv \alpha\left(\left|x_{0}\right| / R\right) / R
$$

Actually, one can strengthen Requardt's theorem and obtain strong convergence (Proposition 3.1), also with a more general time smearing $\alpha_{T(R)}$, which will prove necessary in the charged sectors of QED.

We recall that if $j_{\mu}$ is a Lorentz covariant conserved tempered current, the two point function of the charge density is of the form

$$
<j_{0}(x) j_{0}(y)>=-\Delta J(x-y)
$$

with $J$ a Lorentz invariant tempered distribution of positive type; we denote by $d \nu\left(k^{2}\right)$ the spectral measure defined by $J$.

Proposition 3.1 If the spectral measure d $\nu$ satisfies the (infrared) regularity condition

$$
d \nu\left(k^{2}\right)=k^{2} d \sigma\left(k^{2}\right), \quad d \sigma \text { a measure, }
$$

then, putting $\left.Q_{R, T(R)} \equiv j_{(} f_{R}, \alpha_{T(R)}\right)$ one has

i) $s-\lim _{R \rightarrow \infty} Q_{R, R} \Psi_{0}=0$,

ii) $s-\lim _{R \rightarrow \infty} Q_{R, T(R)} \Psi_{0}=0$ for all functions $T(R)$, with $T(R) / R \rightarrow 0$, satisfying $T(R)>R^{1 / 3}$ and $R \int_{0}^{\varepsilon} d \sigma(s) s /\left(1+T(R)^{2} s^{2}\right)^{2} \rightarrow 0, \varepsilon>0$,

iii) if, for $k^{2} \in[0, \varepsilon), \varepsilon>0, d \sigma\left(k^{2}\right) / d k^{2} \leq C$, the above strong convergence to zero is obtained by choosing $T=R^{1 / 3+\delta}, \delta>0$. 
Proof In fact, one has

$$
\left\|Q_{R, T} \Psi_{0}\right\|^{2}=\int d \nu\left(k^{2}\right) d^{3} q \frac{|\mathbf{q} \tilde{f}(\mathbf{q})|^{2}}{\left.2 \sqrt{(|\mathbf{q}| / R)^{2}+k^{2}}\right)} R\left|\tilde{\alpha}\left(T \sqrt{(|\mathbf{q}| / R)^{2}+k^{2}}\right)\right|^{2} .
$$

Since $\alpha$ is of fast decrease, $\forall N \in \mathbf{N}$,

$$
\left|\tilde{\alpha}\left(T \sqrt{(|\mathbf{q}| / R)^{2}+k^{2}}\right)\right|^{2} \leq \frac{C_{N}}{1+\left((T|\mathbf{q}| / R)^{2}+T^{2} k^{2}\right)^{N}} \leq \frac{C_{N}}{1+\left(T^{2} k^{2}\right)^{N}},
$$

and since $d \nu$ is tempered there is an $M \in \mathbf{N}$ such that $\left(1+k^{2}\right)^{-M} d \sigma\left(k^{2}\right) \equiv$ $d \sigma^{\prime}\left(k^{2}\right)$ is a finite measure. Then, by taking $N=M+2$, one has

$$
\left\|Q_{R, T} \Psi_{0}\right\|^{2} \leq C^{\prime} \frac{R}{T} \int d \sigma^{\prime}\left(s^{2}\right) \frac{T s}{\left(1+T^{2} s^{2}\right)^{2}} \equiv \frac{R}{T} G(T) .
$$

The integrand function is bounded and converges to zero pointwise, when $T \rightarrow \infty$, so that by the dominated convergence theorem $G(T) \rightarrow 0$. Thus i) is proved; moreover strong convergence to zero holds if one chooses $R=$ $T G(T)^{-1+\delta}, \delta>0$ and ii) follows since $\forall \varepsilon>0$,

$$
\int_{\varepsilon}^{\infty} d \sigma^{\prime}\left(k^{2}\right) T \sqrt{k^{2}} /\left(1+T^{2} k^{2}\right)^{2}=O\left(1 / T^{3}\right) .
$$

If the hypothesis of iii) holds one can bound the integral from 0 to $\varepsilon$ by

$$
C \int_{0}^{\varepsilon} d s^{2} \frac{T s}{\left(1+T^{2} s^{2}\right)^{2}} \leq \frac{C}{T^{2}} \int_{0}^{\infty} d u^{2} \frac{u}{\left(1+u^{2}\right)^{2}}=O\left(1 / T^{2}\right) .
$$

Thus, the strong convergence to zero is obtained if $T(R)=R^{1 / 3+\delta}, \delta>0$.

In the physical vacuum sector $\mathcal{H}_{0}$ of QED the assumptions of Proposition 3.1 for the spectral measure of the electric current are satisfied since

$$
<\partial F_{0}(x) \partial F_{0}(y)>=\int k^{2} d \rho\left(k^{2}\right) d^{3} k\left|2 \sqrt{\mathbf{k}^{2}+k^{2}}\right|^{-1} \mathbf{k}^{2} e^{i k(x-y)}, \partial F_{\mu} \equiv \partial^{\nu} F_{\mu \nu},
$$

with $d \rho\left(k^{2}\right)$ the spectral measure of the two point function of $F_{\mu \nu}$. Hence, for $T(R)$ as in ii) of Proposition 3.1,

$$
\lim _{R \rightarrow \infty}\left\|\partial F_{0}\left(f_{R}, \alpha_{T(R)}\right) \Psi_{0}\right\|^{2}=0
$$


and therefore $Q_{R, T(R)}=j_{0}\left(f_{R} \alpha_{T(R)}\right)$ converges strongly to zero on the dense domain $\mathcal{D}_{0}^{p h}$ obtained by applying local bounded observable operators to the vacuum. Eq.(3.2) with $T=R$ was also obtained by D'Emilio [16.

The situation is completely different if one adopts the standard smearing [1, 2], with a fixed $\alpha\left(x_{0}\right)$,

$$
\tilde{Q}_{R}=j_{0}\left(f_{R} \alpha\right) .
$$

Proposition 3.2 The operators $\tilde{Q}_{R}$ have the following properties

i) they converge to zero on $\mathcal{D}_{0}^{p h} \times \mathcal{D}_{0}^{p h}$

ii) $\tilde{Q}_{R} \Psi_{0}$ does not converge weakly in $\mathcal{H}_{0}$, nor does $\tilde{Q}_{R} \Psi, \forall \Psi=U \Psi_{0}, U$ a bounded local operator

iii) there are vectors $\Psi \in \mathcal{H}_{0}$ such that

$$
\lim _{R \rightarrow \infty}<\Psi, \tilde{Q}_{R} \Psi_{0}>
$$

depends on the time smearing test function $\alpha$ (time dependence of the charge)

iv) there are operators $F$ such that,

$$
\lim _{R \rightarrow \infty}<\Psi_{0},\left[\tilde{Q}_{R}, F\right] \Psi_{0}>\neq 0
$$

(Swieca phenomenon [7])

Proof Since in the physical vacuum sector $\tilde{Q}_{R}=((\partial F))\left(f_{R} \alpha\right)$, i) follows by locality and Maison theorem [3].

For ii), the same calculation done above for $Q_{R}$ now gives

$$
\left\|\tilde{Q}_{R} \Psi_{0}\right\|^{2}=R \int k^{2} d \rho\left(k^{2}\right) d^{3} q \frac{|\mathbf{q} \tilde{f}(\mathbf{q})|^{2}}{\left.2 \sqrt{(|\mathbf{q}| / R)^{2}+k^{2}}\right)} R\left|\tilde{\alpha}\left(\sqrt{(|\mathbf{q}| / R)^{2}+k^{2}}\right)\right|^{2},
$$

so that $\tilde{Q}_{R} \Psi_{0}$ cannot converge weakly. Furthermore, $\forall \Psi=U \Psi_{0}$,

$$
\tilde{Q}_{R} U \Psi_{0}=\left[\tilde{Q}_{R}, U\right] \Psi_{0}+U \tilde{Q}_{R} \Psi_{0}
$$

and the first term on the r.h.s converges by locality; since the second term does not converge weakly, neither does the l.h.s.

In order to construct the vector $\Psi$ of iii) we consider

$$
\Psi_{R} \equiv F_{0 i}\left(\left(\partial_{i} \Delta^{-1} g\right) f_{R} h\right) \Psi_{0}, \quad g \in \mathcal{D}\left(\mathbf{R}^{3}\right), h \in \mathcal{D}(\mathbf{R}) .
$$


Such vectors converge strongly to a vector $\Psi \in \mathcal{H}_{0}$, for $R \rightarrow \infty$, since the Fourier transform of $\left(\Delta^{-1} g\right)(\mathbf{x}) h\left(x_{0}\right)$ is square integrable with respect to the measure $d \rho\left(k^{2}\right) d^{3} k\left|k_{0}\right|^{-1}|\mathbf{k}|^{2} k^{2}$ defined by the Fourier transform of $<(\partial F)_{0}(x)(\partial F)_{0}(y)>_{0}$. Then, we have

$$
\begin{aligned}
\lim _{R \rightarrow \infty}<\Psi, \tilde{Q}_{R} \Psi_{0}>= & \lim _{R \rightarrow \infty} \int d \rho\left(k^{2}\right) d^{3} k\left|2 k_{0}\right|^{-1} k^{2} \tilde{f}_{R}(\mathbf{k}) \tilde{\alpha}\left(k_{0}\right) \overline{\tilde{g}}(\mathbf{k}) \overline{\tilde{h}}\left(k_{0}\right) \rightarrow \\
& \overline{\tilde{g}}(0) \int d \rho\left(m^{2}\right) m \tilde{\alpha}(m) \overline{\tilde{h}}(m),
\end{aligned}
$$

which displays the dependence on $\alpha$.

The operators $F_{R} \equiv F_{0 i}\left(\left(\partial_{i} \Delta^{-1} g\right) f_{R} h\right)$ converge strongly to an operator $F$ on the dense domain $\mathcal{A}_{L} \Psi_{0}, \mathcal{A}_{L}=$ the algebra of strictly localized (bounded) observables, since they converge strongly on $\Psi_{0}$ and $\left[F_{R}, A\right], A \in$ $\mathcal{A}_{L}$, becomes independent of $R$, for $R$ sufficiently large by locality. Then, we have

$$
\lim _{R \rightarrow \infty}<\Psi_{0},\left[\tilde{Q}_{R}, F\right] \Psi_{0}>=\overline{\tilde{g}}(0) \int d \rho\left(m^{2}\right) m(\tilde{\alpha}(m) \overline{\tilde{h}}(m)-\tilde{\alpha}(-m) \overline{\tilde{h}}(-m))
$$

which does not vanish in general.

The vector $\Psi$ reflects the infrared behaviour of "dipole states" of the form $\psi_{c}^{\dagger}(f) \psi_{c}(g) \Psi_{0}$, where $\psi_{c}(g)$ is the electron field in the Coulomb gauge, constructed, e.g., according to the Dirac-Symanzik-Steinmann [8, 10] prescription. Thus, in QED, even in the vacuum sector, the naive idea of the charge as the integral of the charge density gives rise to substantial problems because of vacuum polarization effects which disappear only with a suitable time smearing. The same problems arise in the charged sectors of the Coulomb gauge, as stressed by Swieca [7]; they are a general consequence of the non locality of the charged Coulomb fields.

In general, the standard procedure, eq.(2.3), corresponds to taking, in the corresponding correlation functions in momentum space, the limit $\mathbf{k} \rightarrow 0$ and gives a $\delta$ function in $\omega$ only in expectations on local states. On the other hand, Requardt time smearing corresponds to taking a limit $\mathbf{k}, \omega \rightarrow 0$ on the light cone; in expectations on local states, it coincides with that of the standard smearing and it is $\alpha$ independent. As discussed in the Appendix, $\alpha$ independence does not hold on the (non local) charged states of QED and therefore a modification of Requardt's prescription is required for QED. 


\section{Charge density and charge in local formu- lations of QED}

The relation between charge density and charge presents further subtle aspects in the charged sectors. As a consequence of the local Gauss' law, charged states cannot be local. In this section we discuss the limit of the Gauss charges

$$
Q_{R}^{G}=(\partial F)_{0}\left(f_{R} \alpha_{R}\right)
$$

as quadratic forms on local and on physical charged states in the FeynmanGupta-Bleuler formulation of QED and the implications on the possibility of constructing physical state vectors as weak limits of local states.

In the Coulomb gauge, since the charged fields are not local, one has to discuss the limit of local charges on domains obtained from the vacuum by a non local algebra, giving rise to the problems discussed in Sect.2.

Even in perturbation theory the control of the Coulomb gauge is difficult and the standard strategy is to use a local formulation at the expense of positivity; this is the case of the Feynman or Gupta-Bleuler gauge. In this case, the charged fields and the vector potential $A_{\mu}$ are local but their vacuum expectation values cannot satisfy positivity; the corresponding Wightman functions define an indefinite inner product space $\mathcal{D}_{0}=\mathcal{F} \Psi_{0}$, (with $\mathcal{F}$ the local field algebra), with inner product denoted by $\langle.,$.$\rangle , which does not$ contains physical charged states [5, 12].

As suggested by perturbation theory, non local physical charged states may be obtained as suitable limits of local unphysical charged state vectors. A possible non perturbative construction of physical charged state vectors along these lines was discussed in [12].

Quite generally, a crucial issue is that the definition and the control of the limit of local charged state vectors requires a topology; even in the positive case the weak topology on $\mathcal{D}_{0}$ defined by the seminorms $p_{y}(x)=|\langle x, y\rangle|$, i.e. by the Wightman functions is too weak; on the other hand, the inner product space $\mathcal{D}_{0}$ does not identify a unique Hilbert-Krein majorant topology $\tau$ [12] and one has different closures $\mathcal{K}_{\tau}=\overline{\mathcal{D}}_{0}$. For the physical interpretation, the relevant space is the physical subspace $\mathcal{K}_{\tau}^{\prime} \subset \mathcal{K}_{\tau}$, identified by a subsidiary condition (which in QED selects gauge invariant states) and different topologies may give rise to isomorphic physical spaces.

In general, [12] the dependence of the space $\mathcal{K}_{\tau}^{\prime}$ on the topology $\tau$ should not be regarded as a mathematical oddness, since different closures of $\mathcal{D}_{0}$ 
reflect different "boundary conditions" at infinity. Even in the standard theory of unbounded hermitean operators the local domain of $C^{\infty}$ functions of compact support may allow different self adjoint extensions, corresponding to different boundary conditions; in the physical applications the choice of one instead of the other is dictated by physical considerations [12, 17]. In the QED case the lack of non uniqueness reflects the physical fact that different Hilbert-Krein topologies, defined by majorant inner products (., .), correspond to different large distance behaviours of the limit states, classified in particular by the velocity parameter of their Lienard-Wiechert electromagnetic fields al large distances [12. Thus, the choice of the Hilbert-Krein topology is governed by physical considerations since it determines the class of vector states which one can constructively associate to the Wightman functions, i.e. the corresponding closure $\mathcal{K}$ of the vector space $\mathcal{D}_{0}$. For these reasons it should not be a surprise that $\mathcal{D}_{0}$ may allow different extensions. Even in the algebraic approach the construction of the charged states, which correspond to non local morphisms of the algebra of observables, is not under sharp control and in any case does not resolve the multiplicity associated to the large distance behaviour [18.

The choice of the Hilbert-Krein topology in local formulations of QED was discussed at length in [12] also in connection with Zwanziger unsuccessful attempt to construct physical charged states, as a result of a too restrictive Hilbert-Krein topology.

It has been argued [13] that the Gauss charge converges weakly to zero on the local states as a consequence of the vanishing of the Gauss charge commutators with local fields, and that this prevents the construction of physical state with non zero Gauss charge as limits of local states. We shall examine the weak points of this argument in order.

First, the vanishing of the Gauss charge commutators with local fields implies the vanishing of the Gauss charge as a quadratic form on $\mathcal{D}_{0} \times \mathcal{D}_{0}$, (see eq.(2.8) and the Appendix). The vanishing of the Gauss charge on a closure of $\mathcal{D}_{0}$ would follow (see Proposition 4.2 below) if one had weak convergence of $Q_{R}^{G} \mathcal{D}_{0}$ in the topology which defines such a closure of $\mathcal{D}_{0}$.

As we shall see the validity of such a property is not constrained by the correlation functions of the local fields and does not hold in general. Actually, (see the Example below and the following Section) one may find a HilbertKrein topology $\tau$ which avoids the weak convergence of $Q_{R}^{G}$ and allows for the construction of physical charged state vectors.

The failure of the $\tau$-weak convergence of $Q_{R}^{G} \mathcal{D}_{0}$ should not appear strange, 
since it involves a topology whose rôle is merely that of linking the physical non local charged states to the unphysical local states. It should be stressed that the Gauss charge $Q_{R}^{G}$ may well converge weakly or even strongly on a dense domain $\mathcal{D}^{p h}$ of physical states, with respect to the intrinsic Hilbert topology of the physical space. This means that $\forall \Phi \in \mathcal{H}^{p h}, \Psi \in \mathcal{D}^{p h}$, (equivalently $\forall \Phi \in \mathcal{H}^{\prime}, \Psi \in \mathcal{D}^{\prime}$, where $\mathcal{H}^{\prime}$ denotes the distinguished subspace of $\mathcal{K}$ satisfying the subsidiary condition and $\mathcal{D}^{\prime}$ a dense subspace of $\mathcal{H}^{\prime}$ ), one has that

$$
\lim _{R \rightarrow \infty}<\Phi, Q_{R} \Psi>=\lim _{R \rightarrow \infty}<\Phi, Q_{R}^{G} \Psi>, Q_{R} \equiv j_{0}\left(f_{R} \alpha_{R}\right)
$$

exists, equivalently

$$
<Q_{R}^{G} \Psi, Q_{R}^{G} \Psi>=\left\|Q_{R}^{G} \Psi\right\|^{2}
$$

are bounded. This, however, does not mean that $Q_{R} \mathcal{D}^{\prime}$ or $Q_{R}{ }^{G} \mathcal{D}^{\prime}$ converge weakly with respect to the Hilbert-Krein closure $\mathcal{K}$, since weak convergence in $\mathcal{K}$ amounts to the boundedness of

$$
\left\|Q_{R}^{G} \Psi\right\|_{H K}^{2} \equiv\left(Q_{R}^{G} \Psi, Q_{R}^{G} \Psi\right),
$$

where $(.,$.$) is the majorant inner product which defines the Hilbert-Krein$ topology and the corresponding closure $\mathcal{K}$ of the local states $\mathcal{D}_{0}$.

Actually, independently of any Hilbert-Krein majorant, there is a conflict between the construction of the physical charged states in terms of the Wightman functions of the local field algebra $\mathcal{F}$ and the weak convergence of $Q_{R}{ }^{G}$ in the corresponding extension $\mathcal{D}$ of $\mathcal{D}_{0}$. This difficulty is an intrinsic one, since it only involves the Wightman functions of $\mathcal{F}$ and the existence of the physical charged states in an extension $\mathcal{D}$ of $\mathcal{D}_{0}$ compatible with the inner product $<,>$ defined by the Wightman functions, namely such that the sequences of elements of $\mathcal{D}_{0}$ which define the extension, have convergent inner products $\langle$,$\rangle [19]. No reference is needed to a Hilbert-Krein ma-$ jorant topology, even if, clearly, any Hilbert-Krein majorant defines a weak extension. To clarify this point we introduce the following

Definition 4.1 Given two vector spaces $D_{0}$ and $D_{1}$, with inner products $<,>^{(0)}$ and $<,>^{(1)}$, we say that $D_{1}$ can be realized in a weak extension of $D_{0}$ if there exists an inner product vector space $\mathcal{V}$ containing a weakly dense inner product subspaces isomorphic to $D_{0}$ and a subspace isomorphic to $D_{1}$. 
If $D_{0}$ and $D_{1}$ are defined by the vacuum correlation functions of two field algebras $\mathcal{A}_{0}, \mathcal{A}_{1}$, the property of $D_{1}$ being realized in a extension of $D_{0}$ is implied by the existence of joint vacuum correlation functions of $\mathcal{A}_{0}$ and $\mathcal{A}_{1}$. In the case of local formulations of QED, if the correlation functions of the physical field algebra $\mathcal{F}_{1}$, e.g. of the field algebra of the Coulomb gauge, can be constructed in terms of the correlation functions of the local field algebra $\mathcal{F}$, one has an extended field algebra $\mathcal{F}_{\text {ext }}$ generated by $\mathcal{F}$ and $\mathcal{F}_{1}$, and $\mathcal{D}_{1}=\mathcal{F}_{1} \Psi_{0}$ is realized in an extension of $\mathcal{D}_{0}=\mathcal{F} \Psi_{0}$.

Proposition 4.1 Let $D$ be a non degenerate vector space with inner product $<,>, D_{0}$ a weakly dense subspace and $D_{1} \subset D$; let $Q_{R}$ be hermitean charges and

$$
\begin{gathered}
\lim _{R \rightarrow \infty}<D_{0}, Q_{R} D_{0}>=0, \\
\lim _{R \rightarrow \infty}<D_{1}, Q_{R} D_{1}>\neq 0 .
\end{gathered}
$$

Then, $Q_{R} D$ cannot converge in the weak topology defined by $<,>$.

In concrete, if physical charged states $\Psi$ may be obtained as limits of the local states of $\mathcal{D}_{0}$ in a Hilbert-Krein topology $\tau$, i.e. they belong to a (HilbertKrein) extension $\mathcal{D}$ of $\mathcal{D}_{0}$ and

$$
\lim _{R \rightarrow \infty}<\mathcal{D}_{0}, Q_{R}^{G} \Psi>=<\mathcal{D}_{0}, Q \Psi>\neq 0, \quad \lim _{R \rightarrow \infty}<\mathcal{D}_{0}, Q_{R}{ }^{G} \Psi_{0}>=0,
$$

then $Q_{R}{ }^{G} \mathcal{D}_{0}$ cannot converge weakly with respect to $\tau$.

Proof Since $D_{0}$ is dense and $D$ is non degenerate, eq.(4.2) and weak convergence imply that $Q_{R} D_{0}$ converges weakly to zero. Thus

$$
<w-\lim _{R \rightarrow \infty} Q_{R} D, D_{0}>=\lim _{R \rightarrow \infty}<D, Q_{R} D_{0}>=0
$$

and again by the density of $D_{0}, Q_{R} D$ converges weakly to zero, which is incompatible with eq.(4.3).

By eqs.(4.4) and locality $<\mathcal{D}_{0}, Q_{R}{ }^{G} \mathcal{D}_{0}>\rightarrow 0$ and therefore, by the density of $\mathcal{D}_{0}$, weak convergence implies $Q_{R}{ }^{G} \mathcal{D}_{0} \rightarrow 0$ and

$$
<\mathcal{D}_{0}, Q \Psi>=\lim _{R \rightarrow \infty}<\mathcal{D}_{0}, Q_{R}{ }^{G} \Psi>=\lim _{R \rightarrow \infty}<Q_{R}{ }^{G} \mathcal{D}_{0}, \Psi>=0 .
$$

Thus, the construction of physical charged states in a Hilbert-Krein extension of $\mathcal{D}_{0}$ is incompatible with weak convergence of the Gauss charge $Q_{R}^{G}$ on $\mathcal{D}_{0}$. 
The failure of weak convergence of $Q_{R}{ }^{G} \Psi_{0}$ gives rise to the same problems and features discussed in Sect. 2; in particular the domain dependence of the limits of $Q_{R}{ }^{G}$ allows the vanishing of such a limit on $\mathcal{D}_{0} \times \mathcal{D}_{0}$ compatibly with its being non zero on a domain containing non local states (as are the physical charged states).

A Hilbert-Krein topology which allows the construction of physical charged states, avoiding the weak convergence of $Q_{R}{ }^{G} \mathcal{D}_{0}$, was discussed in [12] in terms of the properties of the asymptotic fields $\mathcal{A}_{\mu}^{a s}$. The mechanism is clearly displayed by the following

Example. Let $\psi_{0}$ be a (canonical) free massive Dirac field and $\phi_{1}, \phi_{2}$ two massless scalar fields satisfying the following (equal times) commutation relations

$$
\begin{gathered}
{\left[\phi_{1}, \phi_{2}\right]=0, \quad\left[\pi_{1}, \pi_{2}\right]=0, \quad\left[\phi_{i}, \pi_{i}\right]=0, \quad \pi_{i} \equiv \partial_{0} \phi_{i}, i=1,2} \\
{\left[\pi_{1}(\mathbf{x}), \phi_{2}(\mathbf{y})\right]=\left[\pi_{2}(\mathbf{x}), \phi_{1}(\mathbf{y})\right]=-i \delta(\mathbf{x}-\mathbf{y})}
\end{gathered}
$$

Then, the fields

$$
\begin{gathered}
\phi_{ \pm} \equiv\left(\phi_{1} \pm \phi_{2}\right) / \sqrt{2}, \quad \pi_{ \pm} \equiv\left(\pi_{1} \pm \pi_{2}\right) / \sqrt{2} \\
\psi(x) \equiv U(x) \psi_{0}(x), \quad U(x) \equiv: e^{i \phi_{2}}:(x)
\end{gathered}
$$

satisfy the following commutators and anti commutators

$$
\begin{gathered}
{\left[\phi_{ \pm}(x), \phi_{ \pm}(y)\right]= \pm i D(x-y), \quad\left[\phi_{ \pm}(x), \phi_{\mp}(y)\right]=0,} \\
{\left[\phi_{ \pm}(x), \psi(y)\right]= \pm i D(x-y) \psi(y), \quad\{\psi(x), \bar{\psi}(y)\}=i S(x-y),}
\end{gathered}
$$

where $D, S$ are the standard commutator functions for massless scalar and Dirac fields. Thus, $\phi_{ \pm}$and $\psi$ are local fields.

Our field theory model is defined by the vacuum correlation functions of the field algebra $\mathcal{F}$ generated by $\psi, \phi_{1}$ and $\partial_{\mu} \phi_{2}, \mu=0,1, \ldots 3$ and their Wick products; such correlation functions do not satisfy positivity.

Now, we consider the following local charges

$$
Q_{R}{ }^{\phi} \equiv \partial_{0} \phi_{1}\left(f_{R} \alpha_{R}\right), Q_{R} \equiv j_{0}\left(f_{R} \alpha_{R}\right), Q_{R}{ }^{G} \equiv Q_{R}-Q_{R}{ }^{\phi},
$$

where

$$
j_{\mu}(x)=: \bar{\psi} \gamma_{\mu} \psi:(x)=: \bar{\psi}_{0} \gamma_{\mu} \psi_{0}:(x)
$$


The factorization of the correlation functions of $\psi_{0}$ and $\phi_{ \pm}$implies that $Q_{R}$ converges to an unbroken (non zero) "electron" charge in sense of quadratic forms on $\mathcal{F} \Psi_{0}$ and in fact the correlation functions with unequal numbers of $\psi$ and $\bar{\psi}$ vanish. Actually, $Q_{R} \mathcal{F} \Psi_{0}$ converges strongly with respect to any Hilbert-Krein topology chosen to turn $\mathcal{F} \Psi_{0}$ into a pre-Hilbert space, provided it is a product over fermion and boson Fock spaces since, by positivity of the correlation functions of $\psi_{0}$,

$$
\left\|Q_{R} \Psi_{0}\right\|_{H K}^{2}=<Q_{R} \Psi_{0}, Q_{R} \Psi_{0}>\rightarrow 0 .
$$

The charge $Q_{R}{ }^{G}$ requires a quite different discussion. The field algebra $\mathcal{F}$ is neutral under $Q_{R}{ }^{G}$

$$
\lim _{R \rightarrow \infty}\left[Q_{R}{ }^{G}, \mathcal{F}\right]=0 .
$$

Therefore, putting $\mathcal{D}_{0} \equiv \mathcal{F} \Psi_{0}$, by the argument at the beginning of Sect.2, ii), one has

$$
\lim _{R \rightarrow \infty}<\mathcal{D}_{0}, Q_{R}{ }^{G} \mathcal{D}_{0}>=\lim _{R \rightarrow \infty}<\mathcal{D}_{0}, Q_{R}{ }^{G} \Psi_{0}>=0 .
$$

In the analogy with the local formulation of QED, the local charge $Q_{R}{ }^{G}$ plays the rôle of the Gauss charge, $Q_{R}$ plays the rôle of the electron charge $j_{0}\left(f_{R} \alpha_{R}\right)$ and $Q_{R}{ }^{\phi}$ plays the rôle of the longitudinal charge $\partial_{0} \partial A\left(f_{R} \alpha_{R}\right)$, all smeared in time a la Requardt. As in the QED case the correlation functions of $Q_{R}{ }^{\phi}$ vanish.

The relevant question is whether by taking suitable limits of the local states of $\mathcal{D}_{0}$ one can construct the analog of the physical charged states, i.e. states $\Psi$ satisfying the following condition:

i) positivity, i.e.

$$
<\Psi, \Psi>\geq 0 .
$$

ii) relativistic spectral condition

iii) vanishing expectation of the "longitudinal" field $\partial_{0} \phi_{1}$

$$
<\Psi, \partial_{0} \phi_{1} \Psi>=0
$$

iv) non zero Gauss charge, i.e.

$$
\lim _{R \rightarrow \infty}<\Psi, Q_{R}{ }^{G} \Psi>=\lim _{R}<\Psi, Q_{R} \Psi>\neq 0 .
$$


In the following, such states will be briefly referred to as "physical" charged states.

Similarly to the QED case, the selection of states of $\mathcal{D}_{0}$ satisfying i) - iii) is obtained by means of a supplementary condition

$$
\partial_{0} \phi_{1}^{-} \Psi=0,
$$

which amounts to the exclusion of $\phi_{2}$ components.

As in the QED case, the subspace $\mathcal{D}_{0}^{\prime} \subset \mathcal{D}_{0}$ satisfying the subsidiary condition has zero electric charge; in fact one has $\mathcal{D}_{0}^{\prime}=\mathcal{F}_{0}^{\prime} \Psi_{0}$, where $\mathcal{F}_{0}^{\prime}$ is the field algebra generated by $\phi_{1}$ and by the Wick products

$$
: \bar{\psi} \Gamma \psi:=: \bar{\psi}_{0} \Gamma \psi_{0}:
$$

with $\Gamma$ any element of the algebra generated by the gamma matrices. The problem is whether physical charged states may lie in some completion of $\mathcal{D}_{0}$; as one can easily guess the candidates for the physical states are the free fermion states $\mathcal{A}_{f} \Psi_{0}, \mathcal{A}_{f}=$ the algebra generated by $\psi_{0}$.

If one looks for a Hilbert-Krein completion $\mathcal{K}$ of $\mathcal{D}_{0}$ given by a Krein topology on the boson space, a sufficient condition for $\mathcal{A}_{f} \Psi_{0}$ belonging to $\mathcal{K}$ is that the Hilbert-Krein majorant $($, ) has a Fock structure and, at the level of the two point function $\left\langle\phi_{i} \phi_{j}>, i, j=1,2\right.$, is given by a measure (in $k$ space) of the form

$$
\left(\begin{array}{cl}
|\mathbf{k}|^{2} \beta(|\mathbf{k}|) & 0 \\
0 & |\mathbf{k}|^{-2} \beta(|\mathbf{k}|)^{-1}
\end{array}\right) \frac{d^{3} k}{|\mathbf{k}|},
$$

with

$$
\beta(|\mathbf{k}|) \sim_{|\mathbf{k}| \rightarrow 0}|\mathbf{k}|^{-2 \delta}, \beta(|\mathbf{k}|) \sim_{|\mathbf{k}| \rightarrow \infty}|\mathbf{k}|^{2 \delta}, \quad \delta>0 .
$$

This is in fact the condition which allows the construction of the field $\phi_{2}$ and therefore of $U(x)$ from the derivatives $\partial_{\mu} \phi_{2}$, so that $\psi_{0}$ can be recovered from $\psi$. More generally, the metric leading to a majorization may be chosen independently for each charged sector, i.e. $\beta$ may depend on the charge $q$.

It is instructive to discuss the relation between the existence of charged states and the convergence properties of $Q_{R}^{G}$, which play a crucial rôle in Steinmann argument. First

$$
\left\|Q_{R}^{G} \Psi_{0}\right\|^{2} \equiv<Q_{R}^{G} \Psi_{0}, Q_{R}^{G} \Psi_{0}>\rightarrow 0,
$$


i.e. $s-\lim Q_{R}^{G} \Psi_{0}=0$ in the Hilbert topology defined by the semidefinite Wightman two point function of $j_{0}-\partial_{o} \phi_{1}$, exactly as in the QED case (Sect. 3 , eq.(3.2)).

However, the weak convergence of $Q_{R}{ }^{G} \Psi_{0}$ in $\mathcal{K}$, i.e. with respect to the Hilbert-Krein space to which the physical charged states belong, requires the boundedness of the norm

$$
\begin{gathered}
\left\|Q_{R}{ }^{G} \Psi_{0}\right\|_{H K}^{2}=\left(Q_{R}{ }^{G} \Psi_{0}, Q_{R}{ }^{G} \Psi_{0}\right)= \\
\left.\int d^{3} k|\mathbf{k}|^{-1}|\mathbf{k}|^{2} \beta(|\mathbf{k}|) \tilde{\alpha}\left(R k_{0}\right)\right|^{2}\left|R^{3} \tilde{f}(R \mathbf{k})\right|^{2}= \\
\int d^{3} q|\mathbf{q}| \beta(|\mathbf{q}| / R)|\tilde{\alpha}(|\mathbf{q}|)|^{2}|\tilde{f}(\mathbf{q})|^{2} \sim R^{2 \delta}
\end{gathered}
$$

which requires $\delta \leq 0$. A similar calculation for the weak convergence of $Q_{R}^{G} \psi \Psi_{0}$ in $\mathcal{K}$ involves the choice of the majorization of the boson field correlations in the $q=1$ sector and requires $\delta_{q=1} \leq 0$, whereas the existence of physical states with charge $q=1$ requires $\delta_{q=1}>0$.

In conclusion, in the space $\mathcal{K}$ defined by the above metric with $\delta>0$, there are two dense domains $\mathcal{D}_{0}=\mathcal{F} \Psi_{0}$ and $\mathcal{D}_{1}=\mathcal{F}_{1} \Psi_{0}$, with $\mathcal{F}_{1}$ the field algebra generated by $\psi_{0}$ and by $\phi_{1}, \partial_{\mu} \phi_{2}$, with the properties:

1) $Q_{R}{ }^{G}$ converges to the zero operator on $\mathcal{D}_{0} \times \mathcal{D}_{0}$,

2) $Q_{R}{ }^{G} \Psi_{0}$ converges to zero strongly in the Wightman (semidefinite) scalar product, but it does not converge (even) weakly in the extended space $\mathcal{K}$; moreover $Q_{R}^{G}$ on local charged states does not converge weakly in $\mathcal{K}$

3) $Q_{R}{ }^{G}$ converges to the non zero "electron" charge on $\mathcal{D}_{1} \times \mathcal{D}_{1}$

4) $Q_{R}^{G}$ converges strongly on any vector of $\mathcal{D}_{1}$ satisfying the supplementary condition (eq.(4.11)), in the intrinsic Hilbert topology defined by the Wightman functions.

The model also displays the intrinsic conflict between the construction of the physical charged states and the weak convergence of $Q_{R}{ }^{G}$ in the extended space which contains them; in fact, in the model divergences appear in the limit of matrix elements $<e^{i \phi_{2}} \Psi_{0}, Q_{R}^{G} \Psi_{0}>$. The model also indicates that in QED the Gauss charge converges strongly to the electric charge on a dense domain of physical states (in the intrinsic Hilbert topology of the physical space), a property which is not shared in general by local charges in quantum field theories. 


\section{Comments on the construction of physical charged states}

The construction of physical charged states in local formulations of QED, like the Feynman-Gupta-Bleuler gauge, is a relevant issue because it is strictly related to a non perturbative solution of the infrared problem and provides theoretical support and clarification of the standard perturbative calculations. In Ref. [13], it is argued that physical charged states cannot be obtained as weak limits of the local states, which are at the basis of the perturbative expansion, and that they can only be defined as limits of morphisms of the algebra of observables. The arguments for such a conclusion are on one side the convergence to zero of the Gauss charge in any weak closure of the local states (the weakness of such an argument was discussed in the previous section) and on the other side the divergence of the matrix elements between local states and the physical charged states constructed according to the Dirac-Symanzik-Steinmann (DSS) prescription. In this section we shall critically examine the latter argument and show that a modification of the DSS prescription along the lines discussed in Ref. [12], leads to convergent results for the construction of physical charged state vectors as weak limits of local states.

For this purpose, we adopt the general framework of Ref.[12] and in particular we shall base the discussion on the following assumptions:

I) (existence of asymptotic limits of the vector potential) the asymptotic limits $A_{\mu}^{a s}$, as $=$ in/out, of $A_{\mu}$ exist as (covariant) free fields with the local states in their domains

II) (infrared coherence of "essentially local" states) there are states $\Psi$, in a weak extension of $\mathcal{D}_{0}$, with $\langle\Psi, \Psi\rangle>0$ having a decomposition into (improper) states $\chi^{\alpha}$, with $<\chi^{\alpha}, \chi^{\alpha}>=1$, which are coherent states for $A_{\mu}^{\text {in }}$ (or for $A_{\mu}^{\text {out }}$ )

$$
\begin{gathered}
\left(A_{\mu}^{i n}\right)^{-}(k) \chi^{\alpha}=-\delta\left(k^{2}\right) F_{\mu}^{\alpha,-}(k) \chi^{\alpha}, \\
k^{\mu} F_{\mu}^{\alpha,-}(k)=-e G(k), \quad G^{(0)}=1,
\end{gathered}
$$

with $\left.G^{(} k\right)$ a real symmetric rotationally invariant regular function.

For concreteness, the index $\alpha$, which labels the improper states, can be thought as arising in the direct integral decomposition with respect to the spectrum of the electron momentum $P_{\mu}^{c h}$. For non perturbative and perturbative arguments, which support I) and II), we refer to Ref.[12]. 
We then introduce a function $F_{\mu}^{\alpha}(k)$, with $k F^{\alpha}(k)=$ e sign $k_{0} G(k)$, determined by its restriction $F_{\mu}^{\alpha,-}(k)$ to $C^{-}=\{\mathbf{k},-|\mathbf{k}|\}$ and by the reality condition $F_{\mu}^{\alpha}(k)=\overline{F_{\mu}^{\alpha}(-k)}$, and an operator valued distribution $F_{\mu}(k)$, with

$$
\left[F_{\mu}(k), A^{i n}\right]=0, \quad F_{\mu}^{-}(k) \chi^{\alpha}=F_{\mu}^{\alpha,-}(k) \chi^{\alpha} .
$$

Then, the field

$$
B_{\mu}^{i n}(k) \equiv A_{\mu}^{i n}(k)-\delta\left(k^{2}\right) F_{\mu}(k),
$$

defined on $\mathcal{D}_{\Psi} \equiv \mathcal{A}^{i n} \Psi, \mathcal{A}^{i n}$ the field ${ }^{*}$-algebra generated by $A_{\mu}^{i n}$, satisfies

$$
B_{\mu}^{i n,-}(x) \Psi=0
$$

A physical charged state $\Psi_{p h}$ is then obtained by putting

$$
\Psi_{p h}=e^{i e B_{\mu}^{i n}\left(f^{\mu}\right)} \Psi
$$

provided that the (real) function $f^{\mu}$ satisfies

$$
\delta\left(k^{2}\right) k^{\mu} \tilde{f}_{\mu}(k)=i \delta\left(k^{2}\right) G(k) .
$$

This equation corresponds to the Fourier transform of the Dirac condition $\partial^{\mu} f_{\mu}(x)=\delta^{4}(x)$ restricted to the light cone, since $B^{i n}$ is a free massless field, with ultraviolet regularization provided by $G(k)$. Clearly, all solutions of the Dirac condition $k^{\mu} \tilde{f}_{\mu}(k)=i G(k)$ are also solutions of eq.(5.5).

Eq.(5.5) implies a singularity for $\tilde{f}_{\mu}$ of order at least $1 / k_{0}$ on the light cone and therefore the construction of $\Psi_{p h}$, through eq.(5.4), involves the introduction of an infrared cutoff in $f_{\mu}$. The point is whether its removal can be done in the correlation functions of $e^{i e B_{\mu}^{i n}\left(f^{\mu}\right)}$ and local fields (i.e. Coulomb electron fields exist in the closure of the Gupta-Bleuler space) or only in the expectation of observables on $\Psi_{p h}$.

Since by eq. (5.3) $\Psi$ provides a Fock representation of $B^{a s}$, the existence of $\Psi_{p h}$ in a Hilbert-Krein closure of $\mathcal{D}_{\Psi}$ can be reduced to the finiteness of the two point function

$$
\begin{gathered}
<B_{\mu}^{i n}\left(f_{\mu}\right) \Psi, \eta B_{\nu}^{i n}\left(f^{\nu}\right) \Psi>=\left(B_{\mu}^{i n}\left(f_{\mu}\right) \Psi, B_{\nu}^{i n}\left(f^{\nu}\right) \Psi\right)= \\
\int d^{4} k H^{\mu \nu}(k) \delta\left(k^{2}\right) \overline{\tilde{f}}_{\mu}(k) \tilde{f}_{\nu}(k) \equiv\|\tilde{f}\|_{H K}^{2},
\end{gathered}
$$


where $\eta$ is the operator which defines the corresponding Fock Hilbert-Krein majorant topology. Such a majorization property implies that

$$
\begin{gathered}
\quad|| \tilde{f} \|_{H K}^{2} \geq\left|<B_{\mu}^{i n}\left(f_{\mu}\right) \Psi, B_{\nu}^{i n}\left(f^{\nu}\right) \Psi>\right|= \\
=\left|\int d^{4} k g^{\mu \nu} \delta\left(k^{2}\right) \overline{\tilde{f}}_{\mu}(k) \tilde{f}_{\nu}(k)\right|=|<f, f>|
\end{gathered}
$$

and therefore, in particular, $f_{\mu}$ should be chosen so that the indefinite product $<f, f>$ is finite.

The DSS solution of $k^{\mu} \tilde{r}_{\mu}(k)=i G(k)$, namely $\tilde{r}_{i}(k)=-i k_{i} G(k)|\mathbf{k}|^{-2}$, $\tilde{r}_{0}(k)=0$, does not work, since one obtains

$$
<r, r>=\int d^{3} k|\mathbf{k}|^{-5} k_{i} k_{j} g^{i j} G(k)^{2} / 2,
$$

which is logaritmically divergent for $\mathbf{k} \rightarrow 0$ and therefore, by eq.(5.7), it excludes the convergence of $\|\tilde{f}\|_{H K}$ for any choice of a majorant HilbertKrein topology. This corresponds to the divergence of the two point function $<\Psi_{0}, \psi(x) \bar{\Psi}_{p}(y) \Psi_{0}>$ pointed out by Steinmann (Ref.[13], Ch. 12, p.190).

However, as discussed in Ref. [12], a suitable choice of $f_{\mu}$ avoids the divergence of $\langle f, f\rangle$ and allows for a finite Hilbert-Krein norm.

In fact, all functions of the form $\tilde{f}_{\mu}(k)=\tilde{r}_{\mu}(k)-i k_{\mu} \tilde{g}(\mathbf{k})$ are solutions of eq.(5.5). Since they differ from the DSS solution by a pure gauge, they lead to the same expectations for all observables, but they have different indefinite inner products:

$$
<f, f>=\int d^{3} k G(\mathbf{k})\left(G(\mathbf{k})+2|\mathbf{k}|^{2} \tilde{g}\right)|\mathbf{k}|^{-3} / 2
$$

which vanishes with the choice $\tilde{g}(\mathbf{k})=-|\mathbf{k}|^{-2} G(\mathbf{k}) / 2$. Such a choice gives

$$
\tilde{f}_{\mu}=-i \bar{k}_{\mu}|\mathbf{k}|^{-2} G(\mathbf{k}) / 2, \quad \bar{k} \equiv\left(k_{0},-\mathbf{k}\right) ;
$$

the corresponding operator $A_{\mu}^{i n}\left(f^{\mu}\right)$ describes "zero norm" (unphysical) inphotons and their control depends on the choice of the metric.

The above construction of charged states, based on eqs.(5.4) (5.8), coincides with that of Ref.[12], apart from an infrared convergent gauge term, since in eq.(91) of Ref.[12] for the "infrared dressing" $U$

$$
c_{\mu}(k)=(\sqrt{2}|\mathbf{k}|)^{-1}\left(a k_{\mu}+b \bar{k}_{\mu}\right), \quad a b=1,
$$




$$
\eta d^{+}(f) \eta+d^{+}(f)=(2|\mathbf{k}|)^{-1 / 2} a_{\mu}^{+}\left(k^{\mu} h+\frac{1}{2} \bar{k}^{\mu}|\mathbf{k}|^{-2} G(\mathbf{k})\right),
$$

with $h(\mathbf{k})=O\left(|\mathbf{k}|^{-2+\delta}\right), \delta>0$.

It remains to characterize the conditions on the Hilbert-Krein topology which give $\|\tilde{f}\|_{H K}<\infty$. For this purpose, in the photon $k$-space we introduce four orthogonal four vectors $\varepsilon_{\mu}^{1}(k), \varepsilon_{\mu}^{2}(k), k_{\mu}, \bar{k}_{\mu}$, where $\varepsilon_{\mu}^{1}(k), \varepsilon_{\mu}^{2}(k)$ are (transverse) polarization vectors. Thus, the most general rotation covariant form of $H^{\mu \nu}(k)$ is

$$
H^{\mu \nu}(k)=\beta(|\mathbf{k}|) k^{\mu} k^{\nu} / 2|\mathbf{k}|^{2}+\gamma(|\mathbf{k}|) \bar{k}^{\mu} \bar{k}^{\nu} / 2|\mathbf{k}|^{2}+P^{\mu \nu}(k),
$$

where $P^{\mu \nu}$ denotes the projection on the transverse polarization. Then, since

$$
\sum_{\nu} k^{\nu} k^{\nu}=2|\mathbf{k}|^{2}, \quad \sum_{\nu} H^{\mu \nu}(k) k^{\nu}=\beta(|\mathbf{k}|) k^{\mu}, \quad \sum_{\nu} H^{\mu \nu}(k) \bar{k}^{\nu}=\gamma(|\mathbf{k}|) \bar{k}^{\mu}
$$

positivity of the matrix $H^{\mu \nu}$ requires $\beta, \gamma>0$. Furthermore, since the metric $\eta(k)$ is given by

$$
\left(\eta^{-1}(k)\right)^{\mu \nu}=\sum_{\sigma} g^{\mu \sigma} H^{\sigma \nu}(k)
$$

the condition $\eta^{2}=1$ requires $\beta \gamma=1$. Thus, one gets

$$
\|\tilde{f}\|_{H K}^{2}=\int d^{3} k G(\mathbf{k})^{2}\left(4|\mathbf{k}|^{3} \beta(|\mathbf{k}|)\right)^{-1}
$$

which is finite if $\beta(|\mathbf{k}|) \geq|\mathbf{k}|^{-\delta}, \quad \delta>0$, for $\mathbf{k} \rightarrow 0$. This corresponds to the choice of the metric discussed in the Erice lectures Ref. [12], especially pp. 323, 324, where one can also find a characterization of the metric on the asymptotic fields $A_{\mu}^{i n}$ under general condition on the Fock structure of the representation of $A^{i n}$ given by $\Psi_{p h}$. Weak convergence of the gauge term $\partial_{0} \partial A\left(f_{R} \alpha_{R}\right) \Psi$, which is expected to govern the weak convergence of $Q_{R}^{G} \Psi$, ( $\Psi$ the "essentially local" states at the basis of the construction), would require $\delta \leq 0$, as in the Example of Section 4. In fact, one has

$$
\begin{gathered}
\left\|\partial_{0} \partial A\left(f_{R} \alpha_{R}\right) \Psi\right\|_{H K}^{2}=\left\|\left(B_{R}+C_{R}\right) \Psi\right\|_{H K}^{2}, \quad B_{R} \equiv \partial_{0} \partial B\left(f_{R} \alpha_{R}\right), \\
C_{R} \equiv-e / 2 \int d^{3} q G(\mathbf{q} / R) \tilde{f}(\mathbf{q})[\tilde{\alpha}(|\mathbf{q}|)+\tilde{\alpha}(-|\mathbf{q}|)] .
\end{gathered}
$$

Now, $\left\|C_{R} \Psi\right\|_{H K}^{2}$ remains bounded in $R$ and

$$
\left\|B_{R} \Psi\right\|_{H K}^{2}=\int d^{4} k \theta\left(k_{0}\right) \delta\left(k^{2}\right) k_{0}^{2} H^{\mu \nu}(k) k^{\mu} k^{\nu}\left|\tilde{f}_{R}(\mathbf{k}) \tilde{\alpha}_{R}\left(k_{0}\right)\right|^{2}=
$$




$$
=\int d^{3} q|\mathbf{q}|^{3}|\tilde{f}(\mathbf{q}) \tilde{\alpha}(|\mathbf{q}|)|^{2} \beta(|\mathbf{q}| / R)
$$

diverges if $\delta>0$.

A similar discussion of the choice of the solution of the Dirac condition, can be done for the DSS construction of the physical fields in terms of the local Gupta-Bleuler fields. Again the solution given by eq.(5.8) yields states which differ from the DSS states by a gauge transformation $\exp (i e \partial A(g))$ and by the exponential $\exp (i e[\partial A(g), A(r)])$ of an infrared divergent phase, so that all the expectations of observables coincide with those of the DSS solution. However, it is easy to see that the above phase removes the divergence to order $e^{2}$ of the scalar product $<\Psi_{0}, \psi(x) \bar{\Psi}(y) \Psi_{0}>$, pointed out by Steinmann (Ref. [13], p.190) as an evidence of the claimed impossibility of constructing physical charged state vectors as weak limits of local states.

\section{A Appendix}

In the standard case, locality and unitarity of space time translations imply [3] that, for expectation on local states $\Psi$, eq.(2.8) applies and one has

$$
\begin{gathered}
\lim _{R \rightarrow \infty}\left(\Psi, j_{0}\left(f_{R}, \alpha\right) \Psi\right)=\lim _{R \rightarrow \infty}\left(\Psi, j_{0}\left(f_{R}, \alpha_{R}\right) \Psi\right)= \\
=\lim _{T \rightarrow \infty} \lim _{R \rightarrow \infty}\left(\Psi, j_{0}\left(f_{R}, \alpha_{T}\right) \Psi\right) .
\end{gathered}
$$

Actually, the argument for the vanishing of $\lim _{R \rightarrow \infty}<A j_{0}\left(f_{R}, \alpha\right)>$ only uses locality and the property that the Fourier transforms of $\left\langle A j_{0}(x)>\right.$, $A$ local, are measures. Perturbation theory indicates that this holds in the Feynman-Gupta-Bleuler formulation of QED, where the vanishing of $\lim _{R \rightarrow \infty}<A \partial^{i} F_{i 0}\left(f_{R}, \alpha\right)>$ also follows from the cluster property of the local fields in perturbation theory. For charged states in QED, obtained through a DSS-like construction, one may obtain sufficient localization properties so that the matrix elements $<\Psi_{p h}, j_{0}\left(\mathbf{x}, x_{0}\right) \Psi_{p h}>$ differ by the corresponding elements on local states, in the spacelike complement of a double cone, by corrections of order $|\mathbf{x}|^{-6}$, uniformly in $\left|x_{0}\right|<T_{0}, T_{0}>0$ [20]. However, the matrix elements of $j_{i}$ on such states decrease as $|\mathbf{x}|^{-2}$ [20]:

$$
<\Psi_{p h}, j_{i}\left(\mathbf{x}, x_{0}\right) \Psi_{p h}>=(e / 4 \pi) \int d^{3} z \partial_{i}^{\mathbf{x}}|\mathbf{x}-\mathbf{z}|^{-1} \partial_{0}^{2} K\left(\mathbf{z}, x_{0}\right)+O\left(|\mathbf{x}|^{-4}\right)
$$


where $K$ is the commutator function of the electromagnetic field and eq. (A.1) does not hold for the Gauss charge. In fact, one has

$$
\begin{gathered}
\lim _{R}<\Psi_{p h}, \partial_{0} \partial^{i} F_{i 0}\left(f_{R}, t\right) \Psi_{p h}>=\lim _{R}<\Psi_{p h},-\partial_{i} j_{i}\left(f_{R}, t\right) \Psi_{p h}>= \\
=e \int d^{3} z \partial_{0}^{2} K(\mathbf{z}, t)=-e \int \omega d \omega(\omega \tilde{K})(0, \omega) e^{i \omega t} .
\end{gathered}
$$

The vanishing of the last expression for all $t$ would imply

$$
\omega \tilde{K}(0, \omega)=\lambda \delta(\omega)
$$

and therefore, by Lorentz covariance,

$$
\tilde{K}(k)=\lambda \varepsilon\left(k_{0}\right) \delta\left(k^{2}\right),
$$

i.e. a free theory. Thus, the expectation value of the electric charge, i.e. the electric flux at space infinity, in a charged state defined by Coulomb charged fields is time dependent, even if its time derivative vanishes at $t=0$ (by antisymmetry in $\omega$ ). A current $j_{i}$ with non-zero flux at infinity is therefore present, "induced" by vacuum polarization effects.

The renormalized charge is given by the limit of the matrix elements of the electric flux, with a suitable smearing in time $\left(f_{R}, \alpha_{T}(R)\right.$ as before)

$$
\lim _{R \rightarrow \infty}<\Psi_{p h}, \partial_{i} F_{i 0}\left(f_{R} \alpha_{T(R)} \Psi_{p h}>\right.
$$

In fact, by putting

$$
\tilde{K}(k)=\int d \rho\left(m^{2}\right) \varepsilon\left(k_{0}\right) \delta\left(k^{2}-m^{2}\right),
$$

it follows

$$
\begin{gathered}
\lim _{R \rightarrow \infty}<\Psi_{p h}, \partial_{i} F_{i 0}\left(f_{R} \alpha_{T(R)} \Psi_{p h}>=\right. \\
=e \lim _{R \rightarrow \infty} \int d \rho\left(m^{2}\right) d^{3} k R^{3} \tilde{f}(R \mathbf{k}) \tilde{\alpha}\left(T(R) \sqrt{\mathbf{k}^{2}+m^{2}}\right) \\
=e \int d \mu\left(m^{2}\right) d^{3} q \tilde{f}(\mathbf{q}) \tilde{\alpha}\left((T(R) / R) \sqrt{\mathbf{q}^{2}+R^{2} m^{2}}\right) .
\end{gathered}
$$

Now, for $m^{2}>0,(T(R) / R) \sqrt{\mathbf{q}^{2}+R^{2} m^{2}}>T(R) m$ and $\left(1+m^{2}\right)^{M} \tilde{\alpha}(T m)$ is bounded uniformly in $T$ by a function of fast decrease and converges pointwise to zero. For $m^{2}=0$, the argument of $\tilde{\alpha}$ converges to zero if $T(R) / R \rightarrow 0$ 
and is equal to $|\mathbf{q}|$ if $T=R$. Then, by the dominated convergence theorem, if $T(R) / R \rightarrow 0$ one gets $\lambda e$, with $\lambda$ the $\rho$ measure of the point $m^{2}=0$, which is one by the renormalization condition of the asymptotic electromagnetic field. On the other hand, for $T(R)=R$ one gets

$$
\begin{gathered}
e \lim _{R \rightarrow \infty} \int d^{3} q d \rho\left(m^{2}\right) \tilde{f}(\mathbf{q}) \tilde{\alpha}\left(\sqrt{\mathbf{q}^{2}+R^{2} m^{2}}\right) \\
\quad=\lambda e \int d^{3} k \tilde{\alpha}(|\mathbf{k}|) \tilde{f}(\mathbf{k}) \equiv \lambda e C(\alpha, f),
\end{gathered}
$$

again by the Lebesgue dominated convergence theorem. Thus, Requardt's prescription gives the renormalized charge up to a factor $C(\alpha, f)$. 


\section{References}

[1] C. Orzalesi, Rev. Mod. Phys. 42, 381 (1970)

[2] H. Reeh, Symmetries, Currents and Infinitesimal Generators, Lectures at Haifa 1971, in Statistical Mechanics and Field Theory, R.N. Sen and C. Weil eds., Jerusalem 1972

[3] D. Maison, Nuovo Cim. 11 A, 389 (1972)

[4] M. Requardt, Comm. Math. Phys. 50, 259 (1976)

[5] R. Ferrari, L. Picasso and F. Strocchi, Comm. Math. Phys. 35, 25 (1974); Nuovo Cim. 39 A, 1 (1977)

[6] D. Buchholz, Comm. Math. Phys. 85, 49 (1982); J. Fröhlich, Comm. Math. Phys. 66, 223 (1979); J. Fröhlich, G. Morchio and F. Strocchi, Ann. Phys. 119, 241 (1979)

[7] A.J. Swieca, Nuovo Cim. 52 A, 242 (1967)

[8] K. Symanzik, Lectures on Lagrangean Quantum Field Theory, Desy report T-71/1

[9] P.A.M. Dirac, Canad. J. Phys. 33, 650 (1955)

[10] O. Steinmann, Ann. Phys. 157, 232 (1984)

[11] D. Zwanziger, Phys. Rev. D17, 2570 (1976) and references therein

[12] G. Morchio and F. Strocchi, Nucl. Phys. B211, 471 (1983); 232, 547 (1984); G. Morchio and F. Strocchi, Infrared Problem, Higgs Phenomenon and Long Range Interactions, Lectures at the Erice School, in Fundamental Problems of Gauge Field Theory, G. Velo and A.S. Wightman eds., Plenum Press 1986

[13] O. Steinmann, Perturbative Quantum Electrodynamics and Axiomatic Field theory, Springer 2000, esp. Chs. 7, 12

[14] B. Schroer and P. Stichel. Comm. Math. Phys. 3, 258 (1966)

[15] J.A. Swieca, Goldstone's Theorem and Related Topics, in Cargèse lectures Vol. 4, D. Kastler ed., Gordon and Breach 1969 
[16] E. D’ Emilio, Nuovo Cim. 101A, 757 (1989)

[17] F. Strocchi, Selected Topics on the General Properties of Quantum Field Theory, World Scientific 1993

[18] D. Buchholz, ref. [6]

[19] G. Morchio and F. Strocchi, Representations of *-algebras in indefinite inner product spaces, in Proceedings Conf. on Infinite Dimensional Stochastic Analysis and Quantum Physics, Stochastic Processes, Physics and Geometry: New Interplays. II, Canad. Math. Soc. Conf. Proceedings, Vol. 29, American Mathematical Society 2000, p. 491

[20] D. Buchholz et al., Ann. Phys. 290, 53 (2001) 